\title{
Personally-significant information affects performance only within the focus of attention: a direct manipulation of attention
}

\author{
Assaf Breska • Moran Israel • Keren Maoz • \\ Asher Cohen • Gershon Ben-Shakhar
}

Published online: 4 May 2011

(C) Psychonomic Society, Inc. 2011

\begin{abstract}
It has been suggested that personally significant (PS) information interferes with performance only when presented within the focus of attention. However, this claim was never tested by a systematic manipulation of attention, but only by using correlative measures of its locus. We addressed this issue in two experiments, utilizing a cued visual search paradigm that allowed us to directly manipulate attention and to measure behavioral and physiological responses. One of the stimuli in the search display had a higher luminance value (i.e., was cued), and, orthogonally, one of the stimuli could be a PS or neutral name. When the cue did not predict target location, PS distractors mildly interfered with task performance, regardless of the cue's location. However, when the cue predicted target location, responses were facilitated for cued targets, indicating that attention was shifted to the cue. Importantly, PS distractors interfered with task performance and elicited enhanced orienting responses only when they were cued. This implies that PS information affects performance only when presented within but not outside the focus of attention.
\end{abstract}

Keywords Personally significant stimuli $\cdot$ Task interference $\cdot$ Attention $\cdot$ Visual search $\cdot$ Luminance $\cdot$ Skin conductance response . Orienting response

Much evidence has suggested that attention plays a critical role in tasks involving visual stimuli (e.g., Posner, 1980). Many studies have shown that visual attention operates by selecting targets over distractors (e.g., Yantis \& Johnston, 1990), and a great deal of research has demonstrated that

A. Breska $(\bowtie) \cdot$ M. Israel $\cdot$ K. Maoz $\cdot$ A. Cohen $\cdot$ G. Ben-Shakhar Department of Psychology, The Hebrew University of Jerusalem, P.O. Box 91905, Mount Scopus, Jerusalem, Israel

e-mail: assaf.breska@mail.huji.ac.il even when attention is focused on a specific visual target, certain distractors in the visual field may affect performance (e.g., Kornblum, Hasbroucq, \& Osman, 1990). Put differently, visual attentional control is not perfect, and information not directly relevant to the immediate goals of the task can affect performance. Thus, studies of distraction by unattended stimuli may shed light on the nature of the operation of visual selective attention. One question that arises in this context concerns the types of distractors that affect performance. Ample evidence indicates that task performance is affected by the presence of "task-relevant" stimuli that are not actually required for performing the task, but nonetheless convey task-relevant information (e.g., Stroop, 1935). Importantly, this effect occurs even when such stimuli are presented outside the focus of attention (Cohen \& Shoup, 1997; B. A. Eriksen \& C. W. Eriksen, 1974; Folk, Remington, \& Johnston, 1992). In the present study, we focused on a different kind of distraction, caused by stimuli that are not relevant for the task but convey personally significant information (Gronau, Cohen, \& Ben-Shakhar, 2003). Our goal was to provide direct evidence that PS distractors affect performance only when presented within the central attentional focus. As discussed later, this finding has important implications for the operation of visual attention.

The category of PS information includes all stimuli that have a special meaning to the individual, such as autobiographical items (e.g., the person's first or last name), sometimes referred to as auto-referential stimuli (e.g., Devue \& Brédart, 2008). Historically, this research began with auditory stimuli. The first demonstration that PS stimuli are capable of interfering with the performance of a central task even when they are unattended came from Moray (1959), who used a dichotic listening paradigm. Participants' attention was directed to one auditory channel 
(one ear) by asking them to shadow the content of that channel, while a list of control words was presented to the unattended channel (the other ear). The main finding was that although the words presented to the unattended ear were not remembered, 4 out of 12 participants remembered hearing their own name. This was interpreted as an indication that attention can be captured by unattended PS stimuli. Treisman (1960) suggested that PS words have a lower threshold of activation because they are important for the individual, and consequently they may become activated even when attenuated due to lack of attention.

The initial results of Moray (1959) seemed appealing and fitted well in the context of the historical debate in cognitive science regarding the fate of unattended stimuli. Specifically, this finding seems to constitute a strong argument against early selection theories, such as the influential theory of Broadbent (1958). However, the results reported by Moray have been questioned because the locus of attention in the dichotic listening paradigm was not continuously controlled, and transient wandering of attention between the two auditory channels was possible (Conway, Cowan, \& Bunting, 2001; Lachter, Forster, \& Ruthruff, 2004). Thus, these results do not necessarily reflect unattended processing, but could be explained by attended processing of the name during short periods of shifting attention to the to-be-ignored channel.

Following Moray (1959), others tested the ability of unattended PS stimuli to interfere with the performance of a central task. Wood and Cowan (1995) performed an auditory replication of Moray's study, using various means to increase participants' ability to attend the designated channel and ignore the second channel. Moreover, they monitored the shadowing performance of the participants, in order to detect occasional attention shifts between the two channels. The results of this study were consistent with Moray and revealed that $34.6 \%$ of the participants recalled hearing their name, even under these controlled conditions.

Some studies in the visual domain are also consistent with these findings. Wolford and Morrison (1980) had participants judge whether two digits, presented on both sides of a fixation point, were of the same parity (both odd or both even) or different parities, while a distracting word was presented between the digits. They found a delay in reaction time (RT) to the parity judgment and better memory of the distracting word when it was the participant's name, as compared to a nonname control word. Shapiro, Caldwell, and Sorensen (1997) used the attentional blink paradigm (where it was found that requiring observers to detect a first target impairs the detection of a successive target immediately following it in rapid serial visual presentation; Raymond, Shapiro, \& Arnell, 1992) and demonstrated that when the second target was the participant's name, there was no indication of such a "blink." The same group (Arnell, Shapiro, \& Sorensen, 1999) also found that names were less affected, as compared with other words, by repetition blindness - a failure to notice when a word is repeated during rapid reading. Finally, Mack and Rock (1998) tested the ability of names to overcome inattentional blindness - a failure to notice unexpected stimuli that occasionally appear in an unexpected location when attention is focused on a demanding central task. They found that $88 \%$ of the participants detected the irrelevant stimulus when it was their own name, while only $65 \%$ detected it when it was another first name, and $50 \%$ detected it when it was a frequent English word.

However, other studies found that the presentation of PS stimuli as irrelevant distractors did not impair task performance. Bundesen, Kyllingsbaek, Houmann, and Jensen (1997) presented participants with four names for $150 \mathrm{~ms}$ followed by masking. Two names were colored in red, indicating that they were the to-be-reported targets, while the other two were white. When the participant's name was presented as a target, report accuracy was better than for neutral target names, but importantly, presenting their name as a distractor did not impair performance. Thus, PS information did not capture attention and interfere with task performance in these conditions. Laarni et al. (2000) used a similar paradigm, in which participants had to match two target faces superimposed on an array of task-irrelevant unknown faces, which could in some trials include their own face or a famous face (the president of their country). Laarni et al. found that interference in task performance was similar when participants' own faces and famous faces were presented. In addition, the researchers found that only $18 \%$ of the participants reported noticing their own face in the task display. Finally, Harris, Pashler, and Coburn (2004) tested the ability of PS stimuli to capture attention in a visual search setting. On each trial, participants were presented with an array of words and had to judge whether it contained a previously defined target word. Two of their findings are inconsistent with attention capture by PS names. First, although the participant's name was detected faster than a control name when it was the target, it did not pop out as search time increased with the number of words in the array. More important, when the participant's name was a distractor, it did not interfere with searching for a neutral word more than did a control name. Thus, research in various paradigms revealed that PS stimuli do not necessarily interfere with task performance. This evidence stands in striking disagreement to the previous evidence of attentional capture and interference in task performance by PS stimuli (e.g., Moray, 1959; Wolford \& Morrison, 1980; Wood \& Cowan, 1995).

In recent years, several studies attempted to reconcile these seemingly contradictory findings by examining the conditions under which PS stimuli capture attention in the 
visual domain (e.g., attentional load, Harris \& Pashler, 2004; task set, Kawahara \& Yamada, 2004). Gronau et al. (2003) suggested that the discrepancies between the results of previous studies could be explained by differences in the locus of participants' spatial attention. For example, in the Wolford and Morrison (1980) study, the irrelevant PS distractors always appeared at fixation, suggesting that they could not be completely ignored. On the other hand, in the Bundesen et al. (1997) study, attention was directed to the targets during an early stage of processing, since the cue for the endogenous shifting of attention was color, which is a low-level feature (Folk et al., 1992). Thus, it is possible that attention was not directed to the PS distractors during the trial. Gronau et al. (2003) pointed out that all of the previous findings of distraction by unattended PS stimuli could be explained by lack of stringent control over the locus of attention, allowing for the possibility of occasional wandering of attention to the supposedly unattended channel. To examine this hypothesis, they used a focused attention task in which participants had to classify the color of a target that appeared at fixation, while ignoring a distracting word that could appear either within or outside the focus of attention. Importantly, the word could either be relevant to the task (a color word) or a PS word (the participant's first and last names and the names of parents). In addition to the customary index of interference by RTs, Gronau et al. (2003) measured skin conductance response (SCR), which is a psychophysiological index of the orienting response (OR; see Sokolov, 1963). This component of the OR was initially found following the processing of novel stimuli (see, e.g., Zimny \& Schwabe, 1965), but it is reliably enhanced when processing PS stimuli (e.g., BenShakhar \& Gati, 1987). While task-relevant distractors elicited a congruency effect, when presented both centrally and peripherally, PS distractors affected performance and elicited enhanced ORs only when presented centrally. Later, Devue and Brédart (2008) used a similar manipulation, with the participants' own faces as the PS stimuli, and obtained similar results. Thus, when attention is properly controlled for, PS stimuli that are not task relevant do not interfere with task performance when they appear outside the focus of attention.

The findings of Gronau et al. (2003) and Devue and Brédart (2008) are compelling, yet several important questions remain open. First, these studies did not directly manipulate attention. Instead, the target in their experiments appeared centrally, and the researchers assumed that attention was focused on that location. On the basis of this assumption, stimuli at the target location were defined as appearing within the focus of attention and stimuli that were not part of the target were defined as appearing outside the main focus of attention. Given that attention was not manipulated and there was no evidence of its operation, other factors may account for the difference between the conditions. For example, in the "within attention" condition of the Gronau et al. (2003) study, the distractor (content of word) and the target (the color of the word) were part of the same object, whereas in the peripheral condition the target and distractors belonged to different objects; this difference may have led to the differential effects of the PS stimuli. It is preferable to directly manipulate attention and to observe whether PS stimuli affect performance differently within and outside the attentional focus. Such a manipulation would clarify whether the observed interference reflects the nature of the operation of attentional mechanisms. Bundesen et al. (1997) is probably the only study that has made an attempt in that direction. As described earlier, Bundesen et al. used a red color to direct attention to two of the four display items and found no interference by uncolored PS names. However, this paradigm has several properties that render the conclusions concerning the operation of attention problematic. First, the operation of attention was not explicitly measured. Instead, it was simply assumed that attention was shifted to the relevant color. Second, participants were required to report the words, making them task relevant. Thus, PS and task relevance were dependent. A better-controlled examination of the relations between attention and interference by PS information would require both an explicit indication that attentional selection actually took place and a manipulation of personal significance that was independent of the task.

Second, the conclusions drawn from the results of Gronau et al. (2003) and Devue and Brédart (2008) are valid only for focused attention tasks, where the location of the target is fixed. However, there are other situations in which attention operates in different modes (for an extensive review, see Pashler, 1998). For example, when we seek a desired object whose location is unknown (visual search), we either shift attention across space (Treisman \& Gelade, 1980) or perform a parallel processing of the entire visual field (Duncan \& Humphreys, 1989; Wolfe, Cave \& Franzel, 1989). Evidence from visual search studies has indicated that attention can be captured during the search process either by perceptually salient distractors (Yantis \& Jonides, 1984) or by taskrelevant distractors (Folk et al., 1992; Folk, Remington, \& Wright, 1994). However, the ability of PS distractors to interfere with attentional shifting during the search process when they are task irrelevant was not directly examined. Although the study of Harris et al. (2004) failed to find such interference in search paradigms, its results cannot be clearly attributed to attentional selection because there was no indication that PS names could affect performance under any condition. It is possible that PS stimuli simply do not affect performance in dynamic situations in which attention is "on the move" looking for the target. 
Finally, Gronau et al. (2003) showed a PS effect in both measures (RTs and ORs). However, there are indications that these two measures may tap different processes. Participants are presumably slower when a PS stimulus is present because their attention is prevented from fully focusing on the target. In a similar vein, participants are slower when a target and incongruent distractors are simultaneously present, as in the Stroop and flanker tasks. Yet, while a marked OR is observed for a PS distractor, only a small OR is seen for the Stroop (Gronau et al., 2003) and flanker tasks. Thus, although the OR is an efficient index that the PS stimulus is indeed processed (see Gronau, Ben-Shakhar, \& Cohen, 2005), it is less clear what causes its activation.

The present study directly addressed the first two open questions and indirectly touched on the third. In the present experiments, we used a cued visual search task to directly manipulate attention and examine whether PS stimuli interfere with task performance and capture attention. Moreover, we obtained a direct index for the manipulation of attention and maintained complete independence of the attentional manipulation, the PS manipulation, and the task. In order to further examine the possible differential roles of RT and OR, we measured RTs and accuracy rates, as well as the OR as reflected by SCR.

We used a demanding visual search paradigm in which a target (a string of identical digits) appeared with two types of distractors. One of the distractors was a word that could either be PS or neutral, and the remaining distractors were other strings of identical digits. One of the stimuli (either the target or one of the distractors) had a higher luminance than the rest of the stimuli (hereafter, the singleton). Moreover, the probability that the target would be the singleton was higher than chance, while any one of the remaining stimuli was a singleton on only a small percentage of trials. Since this manipulation made the singleton a predictor of the target's location, we assumed that it would lead participants to actively select the target on the basis of its luminance. Due to this strategy, we assumed that attention would first be directed to the singleton. Importantly, our experimental design enabled us to verify this assumption: If attention is indeed directed first to the illuminated stimulus, the participants' responses should be facilitated in the valid condition (where the target was indeed the singleton) and slower in the invalid condition (where the target was not the singleton). This effect, if found, would validate our assumption that attention is indeed first focused on the singleton. The critical comparison for our main hypothesis is between trials in which the distracting word is PS and trials in which the distracting word is neutral. We predicted a PS effect (i.e., a slower RT and an elevated OR when the distracting word was PS) only on the trials in which the word was the singleton, because in this condition the word was within the focus of attention. There should be no PS effect when the word was not the singleton, and thus was outside the focus of attention. Critically, if a PS effect were to be observed when the target was the singleton, it would contradict our hypothesis, because it would mean that PS interference can occur even when the PS stimulus is positioned outside the focus of attention. Following Gronau et al. (2003), we predicted that such interference would not be observed.

\section{Experiment 1}

Participants in Experiment 1 searched for a target in an array of five stimuli. The array always contained one of two possible targets (a string of the digit 6 or a string of the digit 9 ), three distracting strings of the digit 0 , and a distracting word. Note that this was a difficult task that would normally lead to a serial-like search for the target (see, e.g., Treisman \& Gelade, 1980). There were two main manipulations in the experiment. First, the distracting word could either be neutral or PS. Second, one of the stimuli in the target display had a higher luminance than the others (the singleton). In order to increase the chances that observers would shift their attention to the singleton, we biased the proportions of trials such that the target was the singleton on $50 \%$ of the trials and each of the remaining four stimuli was the singleton on $12.5 \%$ of the trials. As a result, the singleton was a better-than-chance predictor of the target. Numerous studies have shown that this manipulation entices participants to endogenously shift attention to the most likely target (e.g., Jonides, 1981). The probability that the distracting word was personally significant was identical in each of the three luminance conditions, making it independent of the luminance manipulation. To create an effective manipulation of luminance, we used two sequences of displays on each trial, a prime display and a target display. The prime display consisted of five identical strings of the digit 8 . The target display was created out of the prime display by removing one line segment from four of the strings and replacing the fifth string with a word. This prime-target display ensured that the presentation of the target display involved no onsets (i.e., stimuli in positions that had previously been empty), which could have affected the hypothesized attentional effects in the target display (e.g., Rauschenberger, 2003; Yantis \& Jonides, 1984).

If attention were indeed captured by the singleton, three main predictions follow: First, RTs to the target should be faster when it was the singleton, because attention would initially be focused on it. Previous studies (e.g., Gronau et al., 2003; Gronau, Sequerra, Cohen, \& Ben-Shakhar, 2006) indicated that SCR is only minimally affected by task- 
related cognitive conflicts, and thus there should be no effect of the target's luminance on SCR. Second, because attention is directed first to the target when it is the singleton, the distracting word would presumably be outside the focus of attention. Therefore, the content of the word in this condition should not have any effect on either RT or SCR, and we should not obtain any PS effect. Third, and most importantly for our present purposes, a PS effect (slower RTs and elevated SCR to the PS distracting word) should be observed when the distracting word is the singleton. A much lower PS effect should be observed, if any occurs at all, when the singleton is one of the three digit distractors. The reason that a small effect could be expected in this condition has to do with the difficult search required in this task. Once the participant realizes that the singleton (which in this condition is one of the distractors) is not the target, he/she must initiate a search for the target among the remaining four stimuli in the array. During this search, it would be possible that in some proportion of the trials, attention would pass through the distracting word, leading to a PS effect.

The predictions are very different if attention is not affected by the luminance manipulation. In this case, participants should perform a serial-like slow search in all conditions, leading to the following two predictions: First, there should not be any RT or OR differences between the luminance conditions of the target (i.e., whether the target is a singleton or not). Second, a mild PS effect might be observed in all conditions, because the task requires a serial-like search, and attention would be shifted to and focused on the distracting word in some proportion of the trials in all conditions.

\section{Method}

Participants A group of 50 Hebrew University students (37 females, 13 males; mean age 23.4 years) participated in a 40-min experiment for course credit or payment of 20 NIS (about \$5). All participants had normal or corrected-tonormal vision and were native Hebrew speakers. They were briefed about the nature of the SCR measurement and signed an informed consent form.

Apparatus The experiment was conducted in a dimly lit, air-conditioned, and sound-attenuated room. Two $\mathrm{Ag} / \mathrm{AgCl}$ electrodes $(0.8-\mathrm{cm}$ diameter) were filled with a conductive paste (KY Jelly, Johnson \& Johnson, France) and attached to the distal phalanges of the index and fourth fingers of the participant's left hand. Skin conductance was recorded using a standard constant-voltage system of $0.5 \mathrm{~V}$ (DAS-1; Atlas Researchers, Israel), and recordings were continuously digitized by an analog-to-digital converter with a sampling rate of $20 \mathrm{~Hz}$. The SCRs were defined as the maximal conductance incline obtained from 1 to $5 \mathrm{~s}$ after stimulus onset. To minimize motion artifacts, each participant's left hand rested on a chair handrest, and responses were given vocally. RTs were measured using a microphone that was attached to the participant's shirt. Throughout the experiment, an experimenter sat next to the participant and encoded the vocal responses into the computer to keep track of errors. Stimuli were presented on a CRT monitor connected to a PC computer, which also recorded RTs and SCRs. Viewing distance was $80 \mathrm{~cm}$.

Stimuli The stimuli consisted of strings of either letters or digits. The letter strings denoted a meaningful Hebrew word (either a first name or family name). The average letter size was $0.8 \mathrm{~cm}$ in height by $0.7 \mathrm{~cm}$ in width, and the font was David. The digit strings consisted of a repeating digit (e.g., 99999), and across the prime and target displays they could consist of repeated $6 \mathrm{~s}, 9 \mathrm{~s}, 8 \mathrm{~s}$, or $0 \mathrm{~s}$. The digits were presented in a custom-designed font that resembled a 7 segment led display, which allowed for changing the digits from 8 to 6, 9, or 0 by removing one segment (see Fig. 1). The size of the digits was identical to the size of the letters. For each trial, the number of digits in the strings was matched to the number of letters in the letter string presented on that trial. The five strings appeared on a black background (luminosity $0.05 \mathrm{~cd} / \mathrm{m}^{2}$ ) and were placed on the corners of an imaginary equilateral pentagon, with their side character located such that they expanded away from the pentagon center. The pentagon sides were $6 \mathrm{~cm}$, leading to a minimal viewing distance between any two strings of approximately 2.15 degrees of visual angle. The locations of the strings along the circle did not vary between trials. The prime display consisted of five strings of the digit 8 , displayed in a gray color (luminosity $1.27 \mathrm{~cd} / \mathrm{m}^{2}$ ), with the fixation point being a $0.5 \times 0.5 \mathrm{~cm}$ asterisk with the same luminance, presented in the center of the screen. The target display consisted of three distractor strings of the digit 0 , one target string of either $6 \mathrm{~s}$ or $9 \mathrm{~s}$, and a letter string denoting a name. The luminance of four of the strings was identical to that of the prime display, while the luminance of the remaining string (the singleton) was higher (luminosity $42.4 \mathrm{~cd} / \mathrm{m}^{2}$ ).

Design The experimental block included 64 trials. Three variables were manipulated orthogonally within participants: luminance (on target/distracting digit/distracting word), category of the word (first/last name), and personal significance (personally significant name/neutral control name). The target was the singleton on $50 \%$ of the trials, the name was the singleton on $12.5 \%$ of the trials, and one of the three remaining distractors was the singleton on $37.5 \%$ of the trials. Thus, the luminance predicted the location of the target in a greater percentage of the trials than would have been expected by chance $(20 \%)$. Within each of these conditions, the word 
Fig. 1 Experimental stimuli and their probabilities in Experiment 1 , for a participant whose first name is Jason. (Note that word stimuli in the experiment were displayed in Hebrew rather than English.) In all trials, there was a target string of either $9 \mathrm{~s}$ or $6 \mathrm{~s}$ (here, only $9 \mathrm{~s}$ as targets are illustrated), one of the distractors was a name that could be either personally significant or neutral according to the probabilities in the figure (rows), and one of the five stimuli was illuminated according to the probabilities in the figure (columns)
Luminance distribution

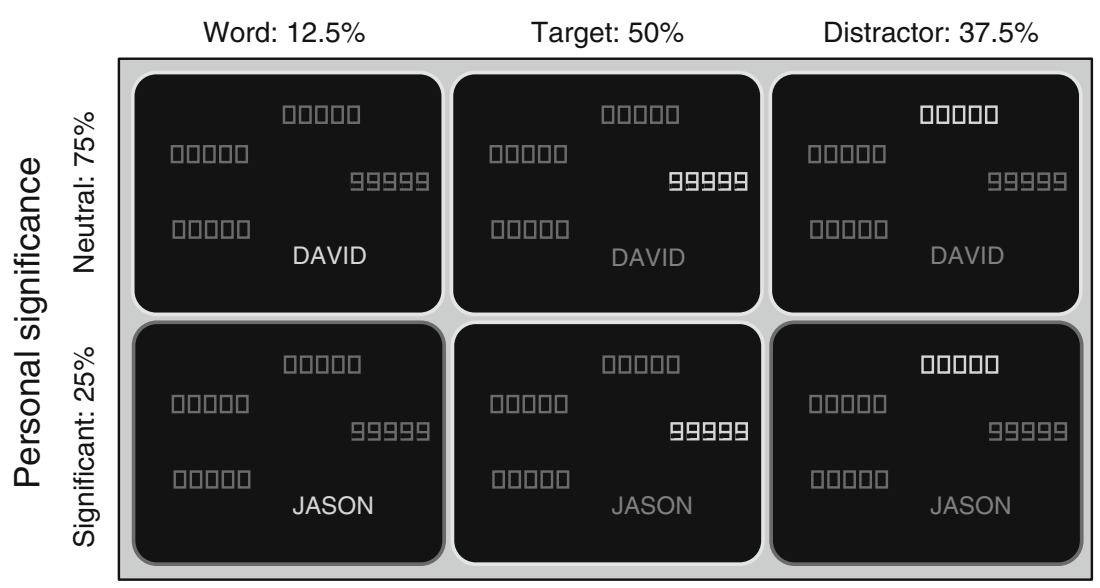

could belong to one of two categories: first name or last name. Within each Category $\times$ Luminance combination, the name was the participant's own first/last name on $25 \%$ of the trials, while in the other $75 \%$ it was a one of three neutral control names, matched in length to the PS name. Within each of these combinations, each target appeared once. The locations of the target and the name distractor were randomized between trials such that, across the entire block, they appeared at approximately equal frequencies at each of the five possible locations, and there were equal probabilities for them to be adjacent or spaced by one distractor. The target factor was not manipulated orthogonally to the other factors, because this would have required too many trials. Instead, the targets were pseudorandomly assigned to the different trials, with the following constraints: Within each participant, the identity of the target was evenly distributed across trials (i.e., on half of the trials the target was a string of $6 \mathrm{~s}$, and on the other half it was a string of $9 \mathrm{~s}$ ). In addition, they were also evenly distributed in all of the relevant word conditions (when the word was illuminated, when a PS word was present, and when a PS word was illuminated).

Procedure Prior to the beginning of the experiment, participants supplied a list of names of all of their siblings and an additional 10 familiar names that were disqualified from being used as distractors. The participants were seated facing the computer screen; the electrodes were attached to their fingers and the microphone to their shirt. They were instructed to find the string that contained either $6 \mathrm{~s}$ or $9 \mathrm{~s}$ and to name the digit as quickly as they could. They were also instructed to find a comfortable posture that would enable them to minimize any movements and to say nothing but "six" or "nine." The instructions included no explicit reference to the luminance. Participants then performed a practice block of 16 trials with an ITI of $1 \mathrm{~s}$, which included only nonname words (e.g., lamp, chair), followed by an additional 3 practice trials with the long experimental ITI (see below) in order to accustom them to the longer intervals. After the practice trials, a 2-min baseline measurement of skin conductance level at rest was conducted, after which the experiment began. The 64 experimental trials were presented at varying ITIs of 16-24 s (mean $20 \mathrm{~s}$ ), with a short break after 32 trials. At the beginning of the experimental block and after the break, a buffer trial that included a neutral word was presented. The buffer trials, which were not taken into account in the data analyses, were used because the presentation of the first stimulus usually elicits a large OR. On each trial, the prime display was presented for $500 \mathrm{~ms}$ and then switched to the target display, which remained on screen until a vocal response was detected. After the end of the experimental block, the participants were released from the electrodes and microphone, debriefed, and paid.

\section{Results}

Trials on which the RT was below $200 \mathrm{~ms}$ or above 2,000 ms, error trials, and trials on which the response was other than naming the digit were eliminated from the analysis $(2.7 \%)$. Trials on which participants showed any behaviors that could have elicited an OR (movements, sneeze, etc.) during the SCR measurement window were discarded from the SCR analysis only (additional $2.8 \%$ ). The proportions of errors were very small and did not differ between the conditions ( $\sim 0.1 \%$ within each condition), and therefore these were not analyzed further. For each measure of each participant, responses to trials within each Luminance $\times$ Category $\times$ Personal Significance $\times$ Target combination were averaged and subjected to a $3 \times 2 \times 2 \times 2$ repeated measures ANOVA. The results are reported across word categories and targets, as these factors showed neither statistically significant main effects nor interactions with any of the factors of interest, and were evenly distributed across them.

Figure 2 presents mean RTs and SCRs for each Luminance $\times$ Personal Significance combination across participants, and Table 1 presents a summary of the $3 \times 2$ 


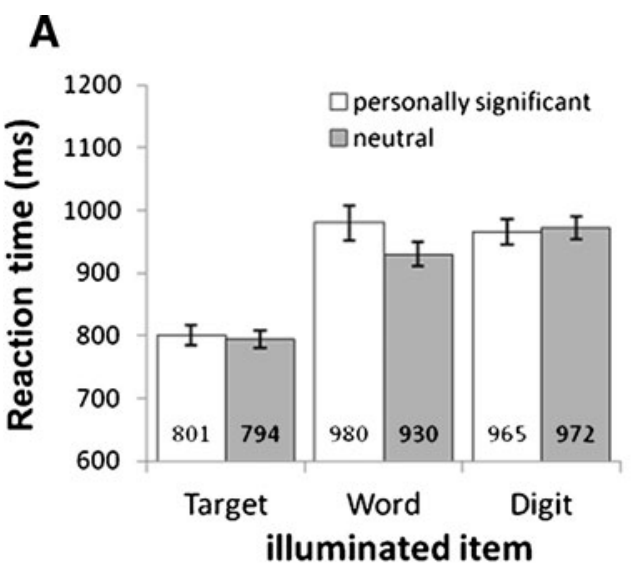

B

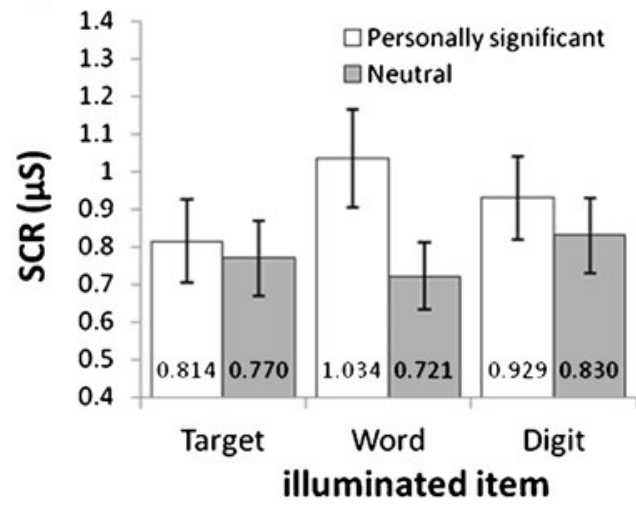

Fig. 2 Mean reaction times (a) and skin conductance responses (SCRs) (b) in Experiment 1, computed separately for conditions in which the luminance cue was on the target, the irrelevant name, or an irrelevant distractor, and for conditions in which the name was personally significant or neutral

ANOVA on these two factors. We first examine the success of the luminance manipulation. The pattern of the RT results indicates that participants were much faster when the target was the singleton $(M=797)$ than when one of the distractors was the singleton (word singleton, $M=955$; digit singleton, $M=968$ ). A planned contrast revealed that this effect resulted from shorter RTs when the target was illuminated than when the distractors were illuminated $[F(1,49)=78.08, M S E=17,318, p<.001$, Cohen's $f=0.88]$. This robust finding indicates that the luminance manipulation was successful in directing attention to the singleton. In accord with our previous findings concerning the congruency effect in the Stroop and flanker tasks (e.g., Gronau, Cohen, \& Ben-Shakhar, 2009), the SCR measure showed little sensitivity to the attentional manipulation.

Given that attention was indeed captured by the luminance manipulation, we focused on the pattern of the PS effect. Overall, RTs were significantly longer for PS names than for neutral names, with a marginally significant interaction with the Luminance factor. A planned interaction contrast in which we compared the PS effect when the word was the singleton to the PS effect when the target or a distractors was the singleton was statistically significant $[F(1,49)=4.13, M S E=7,572, p<.05$, Cohen's $f=0.13]$. Separate analyses of the PS effect within each of the three luminance conditions revealed, as predicted, that when the word was the singleton, RTs for the PS name were longer than those for the control names. (The mean PS effect was $49.9 \mathrm{~ms}$, with a $95 \%$ confidence interval of $5.2-94.5 \mathrm{~ms}$ ). This effect was statistically significant $[t(49)=2.24, p<.05$, Cohen's $d=0.32]$. In contrast, when the target was the singleton, RTs for the PS names did not differ from RTs for the control names $[t(49)=0.78, p>.4$; mean PS effect $=6.7 \mathrm{~ms}, 95 \%$ confidence interval $=-10.6$ to 24]. Likewise, when the singleton was one of the digit distractors, no PS effect was obtained. In fact, the mean RT for the PS word in this condition was somewhat smaller than that for the neutral word $[t(49)=0.424, p>.5$; mean PS effect $=-7 \mathrm{~ms}, 95 \%$ confidence interval $=-40$ to 26.1$]$.

SCRs were also significantly larger for PS names than for neutral names, with a significant interaction with the Luminance factor. We performed a planned interaction contrast identical to the one conducted for RTs and found that the PS effect was significantly larger when the word was the singleton than when a distractor or the target was the singleton $[F(1,49)=6.22, M S E=0.118, p<.05$, Cohen's $f=0.16]$. Separate analyses of the PS effect for each of the three luminance conditions revealed that, in accord with our hypothesis, SCRs to the PS names were larger than those for the control names when the word was the singleton $[t(49)=3.49, p<.01$, Cohen's $d=0.5$; mean PS effect $=0.31,95 \%$ confidence interval $=0.13$ to 0.49 ] When the target was the singleton, SCRs to the PS and control names did not differ $[t(49)=1.05, p>.1$; mean PS effect $=0.045,95 \%$ confidence interval $=-0.04$ to 0.13 ] Interestingly, when one of the distractors was the singleton, SCRs to the PS names were somewhat larger than those to the control names, with this effect being marginally significant $[t(49)=1.9, p=.064$, Cohen's $d=0.27$; mean PS effect $=0.10,95 \%$ confidence interval $=-0.01$ to 0.20 ].

Control experiment To ensure that the effects observed in Experiment 1 were indeed caused by the luminance manipulation, we conducted a control experiment in which each stimulus in the array could be the singleton with an equal probability. Thus, in this experiment, the singleton did not predict target location. We examined the pattern of the PS effect under these conditions. A total of 32 students (11 females, 21 males; mean age 23.8 years) participated in this experiment, which was identical to Experiment 1, except that each of the five display items was the singleton in $20 \%$ of the trials. We predicted that no difference in overall performance between the luminance conditions would be obtained. In addition, because the search in our task was effortful and probably required a serial search, we 
Table 1 Full ANOVA table (with the factors Luminance and Personal Significance) of Experiment 1

${ }^{*} p<.001 .{ }^{* *} p<.05 .{ }^{* * *} p=.057$.

predicted a general mild PS effect across all conditions, with no interaction with the Luminance factor.

There were no significant RT differences between trials on which the target was the singleton and trials on which one of the distractors was the singleton. This indicates that in this setting, attention was not captured by the singleton due solely to its luminance saliency. Moreover, under these conditions, there was a mild but statistically significant PS effect in both measures in all luminance conditions, including the target-illuminated condition. This finding stands in contrast to the lack of a PS effect in the targetilluminated condition of Experiment 1, and it indicates that this does not reflect an inability of the words to capture attention under the present display parameters. Rather, it implies that the lack of a PS effect in Experiment 1 resulted from lack of attention to the word when the target was illuminated.

\section{Discussion}

Several conclusions can be drawn from the pattern of results observed in Experiment 1. First, the luminance manipulation succeeded in triggering an attentional shift toward the singleton. Most relevant for the present purposes, when the word was the singleton, performance was impaired and SCRs were appreciably elevated when presenting a PS name as compared to a control name, pointing to a clear PS effect. This finding supports our hypothesis that PS stimuli affect performance when presented within the focus of attention. The lack of differences in RTs and SCRs between the PS and the neutral words when the target was the singleton indicates, in line with our hypothesis, that PS stimuli do not interfere with task performance and do not elicit an OR when positioned outside the main attentional focus. The results of the control experiment indicate that this finding cannot be explained by factors other than the lack of attention to the PS stimulus.

While these results support our main hypothesis, some of the findings are less clear. Specifically, when one of the digit distractors was the singleton, there was a tendency toward increased SCRs to the PS words, but with no parallel effect for the RT measure. How can these seemingly inconsistent results be accounted for? Consider the processes that must take place when the singleton is a digit distractor, assuming that attention is first directed to the singleton: Once participants realize that the singleton is not the target, they have to initiate a serial-like search among the remaining stimuli in the array. ${ }^{1}$ On some percentage of these trials, the word distractor might fall within the focus of attention, and on those trials larger SCRs would be elicited. If so, the small PS effect observed when one of the digit distractors was illuminated, as well as the PS effect observed in the control experiment, occurs when the name emerges within the focus of attention. This effect must be due to postcapture search processes, because it was not observed when the target was the singleton, in which case no postcapture search was required. ${ }^{2}$

If the PS effect observed with the SCR measure reflects postcapture processing of the PS word, why was no

\footnotetext{
${ }^{1}$ The present paradigm does not allow us to conclude whether the postcapture search processes are serial in nature (Treisman \& Gelade, 1980) or reflect the operation of a limited-capacity parallel processing system (Bundesen, 1990; Eriksen \& Yeh, 1985). However, this distinction is irrelevant for the interpretation of the present results, because according to both possibilities some attention is directed to the PS name, making it within the focus of attention at some point during the trial. We thank an anonymous reviewer for pointing out the possibility of parallel search.

${ }^{2}$ If indeed the PS effect observed with the SCR when a digit distractor was the singleton reflects postcapture processes, we would expect a positive correlation between the time spent on the PS word and the SCR elicited by this word. Under this explanation, no such correlation would be expected when the target is illuminated, because in this condition attention is shifted directly to the target and the word is never in the attentional focus. Accordingly, we calculated for each participant the correlation between the RTs and SCRs across trials in which a digit distractor was illuminated and the word was PS. The mean correlation was significantly larger than $0[r=.16 ; t(49)=2.45$, $p<.05]$. When the target was illuminated, the mean correlation was smaller and not significantly larger than $0[r=.08 ; t(49)=1.42, p>$ .1]. Furthermore, a sign test revealed that these correlations were significantly larger when the digit distractor was the singleton than when the target was illuminated ( $z=1.69, p<.05$, one-tailed).
} 
counterpart observed with the RT measure? There are at least two possible explanations: One possibility is that the SCR measure is simply more sensitive to the PS effect (cf. Gronau et al., 2005). Another possible explanation concerns a fundamental difference between these two measures. As described earlier, whereas the RT measure reflects attentional processes, the SCR may reflect other processes associated with PS information. It is possible that following the capture of attention by the digit distractor, participants search the array exhaustively (i.e., scanning the entire array before making a decision). If so, RTs would not be affected by the PS distractor. Although there is no systematic documentation of such a strategy for visual search, exhaustive search has been extensively documented in the memory search literature (e.g., Sternberg, 1969). In contrast, OR is activated as soon as the PS stimulus is processed, leading to an increased SCR. The goal of Experiment 2 was to clarify these issues by providing more evidence for our hypothesis that the PS effect obtained when the digit distractor was illuminated was due to postcapture search that took place once the participant realized that the singleton was not the target.

\section{Experiment 2}

Experiment 2 was essentially the same as Experiment 1, except for one change: The target display was not terminated by the response, as in the previous experiments. Instead, the target display was presented for $500 \mathrm{~ms}$, and was then masked by a display of hashes (\#) that stayed on the screen for another $500 \mathrm{~ms}$. This manipulation severely limited the possibility of a search when the singleton cue was invalid. Thus, the masking would ensure that any SCRbased effect must be due to events that took place prior to the masking. We used an SOA of $500 \mathrm{~ms}$ because the mean RT in Experiment 1 when the target was the singleton (and therefore no postcapture search was needed) was around $800 \mathrm{~ms}$. Therefore, we assumed that an exposure time of $500 \mathrm{~ms}$ would make the task far more difficult for the participants and, at the very least, significantly reduce the time left for the postcapture search. Consequently, the SCR effects observed in Experiment 1 when the distracting digits were singletons should be reduced, and possibly completely eliminated, in Experiment 2.

This design has a clear drawback with respect to the RT measure. As observed in Experiment 1, RT was not sensitive to the PS word once a (postcapture) search was initiated. The masking in the present design leads to a much more difficult task in which participants would focus much more on being accurate. Specifically, while the singleton is still a good predictor of the target and is still beneficial to first search for it, participants might develop strategies for quickly disengaging from the singleton in the invalid trials that would mask RT-based PS effects in these invalid conditions. Our main predictions in this experiment, therefore, concern the SCR effects. With this measure, we predicted that a pronounced PS effect would be obtained when the word was the singleton, and no effect would be observed when the target or the distracting digits were the singleton.

\section{Method}

Participants Another group of 50 Hebrew University students (23 females, 27 males; mean age 23.7 years) participated in a 40-min experiment for course credit or a payment of 20 NIS (approximately \$5). All participants had normal or corrected-to-normal vision and were native Hebrew speakers. They were briefed about the nature of the SCR measurement and signed an informed consent form.

Stimuli and design The experimental stimuli and design were identical to those of Experiment 1. However, in addition to the prime and target displays, there was also a masking display, which consisted of five strings containing hashes/number signs, appearing in the same locations as the five stimuli in the prime and target displays. The size of each of the hashes was slightly larger than that of the individual letters, $0.9 \times 0.8 \mathrm{~cm}$, and they were presented at the same level of luminance $(1.27 \mathrm{~cd} / \mathrm{m} 2)$ as the stimuli in the prime display (and all of the stimuli but the singleton in the target display).

Procedure The procedure was identical to that of Experiment 1, except that on each trial, the target display appeared for $500 \mathrm{~ms}$ and was then immediately replaced by the masking display, which also appeared on the screen for $500 \mathrm{~ms}$. Participants were given the same instructions, but were also informed that the target display would appear for a very short time and that they should do their best to find the target. It was emphasized that even if they failed to locate the target and identify it, they should still respond as fast as they could, based on what they managed to perceive from the display. This was done to make sure that a vocal response would be uttered on all trials.

\section{Results and discussion}

As in Experiment 1, outliers (2.5\%) were eliminated from the RT analysis. As expected, error rates were much higher than in Experiment 1. Erroneous trials were discarded from the RT and SCR analyses (13.7\%). Given the higher percentage of errors in this experiment, differences between 
conditions on this measure were analyzed as well. Trials with OR-eliciting behaviors during the SCR measurement window were discarded from the SCR analysis (additional $2.8 \%$ ). For each participant, RTs, SCRs, and error rates were averaged within each Luminance $\times$ Category $\times$ Personal Significance $\times$ Target combination and subjected to a $3 \times 2 \times 2 \times 2$ repeated measures ANOVA. Again, the results are reported across categories and targets, since these factors had no significant main or interaction effects with the other variables.

Figure 3 presents the mean RTs, SCRs, and error rates for each of the Luminance $\times$ Personal Significance combinations, and Table 2 presents a summary of a $3 \times 2$ ANOVA on these two factors. In this experiment, we focused primarily on the SCR results. PS names elicited significantly larger SCRs $(M=0.753)$ than control names $(M=0.671)$. Importantly, the Luminance $\times$ Personal Significance interaction was also significant. Planned comparisons revealed that the PS names elicited larger SCRs than did the control names when the word was the singleton $[t(49)=3.63, p<.001$, Cohen's $d=0.52$; mean PS effect $=0.29,95 \%$ confidence interval $=0.13$ to 0.46 ], but not when the target was the singleton $[t(49)=1.14, p>$ .1 ; mean PS effect $=0.04,95 \%$ confidence interval $=-0.03$ to 0.11 ]. Importantly, when one of the distracting digits was the singleton, the SCRs were insignificantly smaller for PS names than for neutral names (mean PS effect $=0.09,95 \%$ confidence interval $=-0.176$ to 0.001 ). To directly compare the two experiments with respect to the PS effect obtained in this condition, we performed a $2 \times 2$ mixed ANOVA (Exp. 1 vs. Exp. $2 \times$ PS vs. neutral words), which yielded a significant interaction $[F(1,98)=7.48, M S E=0.058, p<$ $.01]$. These findings are fully consistent with the hypothesis that PS stimuli affect SCRs within but not outside the attentional focus.

For the RT measure, responses were faster when the target was the singleton than when one of the distractors was illuminated. As in Experiment 1, this finding clearly indicates that participants searched first for the singleton. Neither the PS main effect nor its interaction with the Luminance factor produced statistically significant effects. Planned contrasts comparing the differences between the PS and neutral names within each luminance condition did not reveal any statistically significant effects (all $p \mathrm{~s}>.05$ ).

Figure 3 also displays error rates for each condition. The error rates were calculated across participants, because within each participant the number of trials in certain conditions was too small to obtain a reliable estimator of the percentage of errors. There were fewer errors in the target luminance condition $(6.8 \%)$ than in the other conditions $\left[21.1 \% ; \chi^{2}(1)=134.14, p<.001\right]$, further supporting the assumption that attention was directed first to the singleton. However, there was no difference in error



B
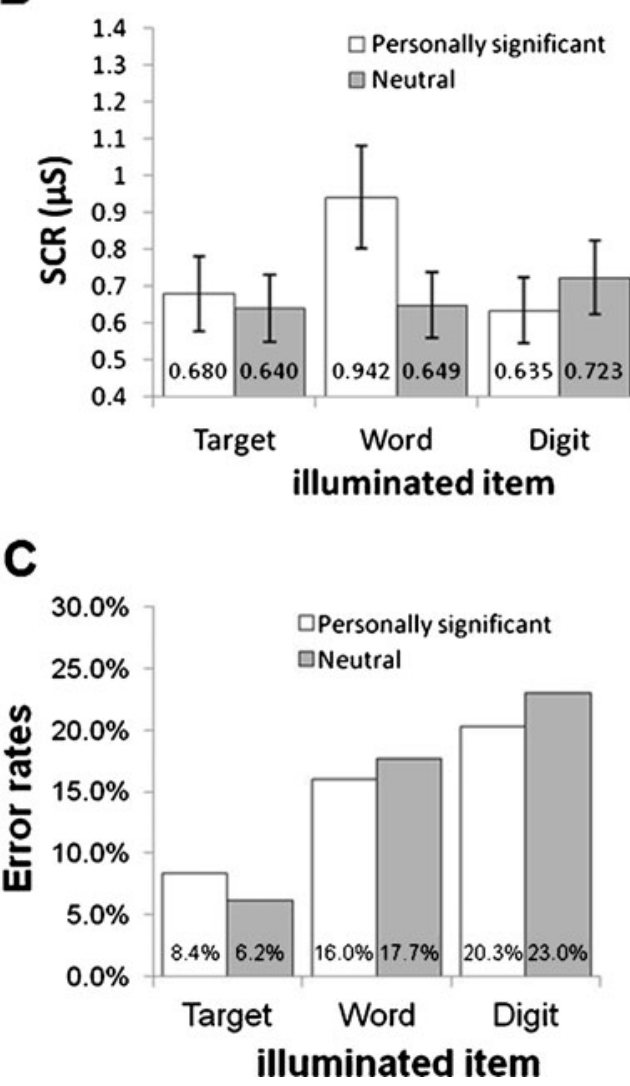

Fig. 3 Mean reaction times (a), skin conductance responses (SCRs) (b), and error rates (c) in Experiment 2, computed separately for conditions in which the luminance cue was on the target, the irrelevant name, or an irrelevant distractor, and for conditions in which the name was personally significant or neutral

rates between the PS and control names $\left[\chi^{2}(1)=0.15, p>\right.$ .1]. In addition, planned comparisons revealed that there were no significant differences in error rates between the PS and control names within each luminance condition [word, $\chi^{2}(1)=0.16, p>.1$; target, $\chi^{2}(1)=2.29, p>.1$; distractor, $\left.\chi^{2}(1)=0.94, p>.1\right]$.

The results of Experiment 2 reveal that, in contrast to the results of Experiment 1, the PS effect with SCRs was observed exclusively when the word distractor was the 
Table 2 Full ANOVA table (with the factors Luminance and Personal Significance) of Experiment 2

\begin{tabular}{lllll}
\hline & $F$ Value & $d f$ & $M S E$ & Cohen's $f$ \\
\hline Reaction Times & & & & \\
$\quad$ Luminance & $23.34^{*}$ & 2,98 & 42,016 & 0.39 \\
Personal Significance & 1.33 & 1,49 & 16,851 & \\
Luminance x Personal Significance & 0.10 & 2,98 & 20,992 & \\
Skin Conductance Responses & & & & \\
Luminance & $4.74^{* *}$ & 2,98 & 0.114 & 0.14 \\
Personal Significance & $5.33^{* *}$ & 1,49 & 0.094 & 0.12 \\
Luminance x Personal Significance & $12.66^{*}$ & 2,98 & 0.074 & 0.28 \\
\hline
\end{tabular}

singleton. The combined results of the two experiments suggest that a major part of the mild SCR effect in Experiment 1 is due to processes that take place after the distracting digit captured attention. This pattern of results was not observed in Experiment 2 because the masking prevented postcapture processes from taking place.

Another difference between Experiments 1 and 2 concerned the PS effects as measured by RTs. The PS effect in Experiment 1 was reflected by both measures. In contrast, the PS effect in Experiment 2 was observed only with the SCR measure. Recall that the RT measure was also not sensitive to PS stimuli during the postcapture search of Experiment 1. As mentioned before, there are at least two possible explanations for the lack of an RT effect. One possibility is that the RT measure for PS stimuli is less sensitive than the SCR measure. Alternatively, the result might be due to the difficult situation facing the participants in the invalid condition, which may have led them to use an exhaustive search strategy that would mask any RT effect. Admittedly, both are ad hoc explanations and require further research.

\section{General discussion}

In this study, we examined the ability of personally significant but task-irrelevant distractors to interfere with the performance of a visual task. Gronau et al. (2003) and Devue and Brédart (2008) suggested that such stimuli affect performance within but not outside the focus of attention. Yet, no study to date had directly manipulated attention to test this hypothesis. In the reported experiments, we explicitly manipulated attention and provided support for this hypothesis. Specifically, we used a cued visual search paradigm in which one of the search items was illuminated. Because the target was illuminated more frequently than each of the distractors, it was profitable for observers to follow this cue (cf. Jonides, 1981). The results of Experiment 1 strongly indicated that this luminance manipulation captured attention. The search array also contained a word that could be either PS or neutral, orthogonally to the validity of the cue. As expected, we found a PS effect when the singleton was the word distractor, but not when it was the target.

Unexpectedly, however, when one of the digit distractors was the singleton in Experiment 1, a marginal PS effect was reflected by the SCR. Note that the luminance manipulation only ensured that the initial focus of attention would be on the singleton. There was no control on the locus of attention once the participant realized that the singleton was not the target. We hypothesized that the marginal SCR effect observed in this condition reflected a postcapture search, whereby participants briefly focused on the word on some of the trials. This hypothesis was supported by a post-hoc analysis of the correlation between RTs and SCRs (see note 2) and by the results of Experiment 2, which was designed to directly examine this hypothesis. In Experiment 2, we used a masked display that limited and possibly excluded postcapture processes. In accord with our hypothesis, an SCR-based PS effect was obtained exclusively for the trials on which the singleton was the word distractor.

Another notable difference between Experiments 1 and 2 is the pattern of results with the RT measure. Whereas participants were slower in Experiment 1 when the PS word was the singleton, the masking procedure in Experiment 2 eliminated the RT effect altogether. This finding supports the claim that OR and RT measures tap fundamentally different processes. The OR measure does not reflect attentional processes. For example, the OR is minimally affected by interference tasks such as the flanker and Stroop tasks (e.g., Gronau et al., 2003, 2009). The PS effect as measured by the OR may reflect the registration of important as well as unexpected information. In contrast, the RT measure is sensitive to attentional processes and is therefore larger when attention is delayed by a distractor (as is the case in interference tasks such as the flanker and Stroop tasks). The RT results of Experiments 1 and 2 suggest that attentional processes were slowed by the PS distractor in Experiment 1 but not in Experiment 2. We suggest that this difference in the search processes between Experiment 1 and 2 stems from the different demands imposed by the task in the two experiments. In particular, 
the masking procedure in Experiment 2 induced a highly demanding challenge. On trials on which the singleton was not the target, participants had to switch their attention very quickly to the remaining distractors under a severe time pressure. This context may have induced participants to employ an exhaustive search (cf. Sternberg, 1969) that eliminated the RT difference between the conditions.

Although the explanation for the different RT results obtained in the two experiments may seem ad hoc, other studies are compatible with this explanation. Numerous studies in the memory search literature (see Sternberg, 1969, for a review) have shown that participants may use exhaustive search when the context calls for it, and this strategy may be used in visual search tasks similar to those used in this study. For example, as we reviewed earlier, Harris et al. (2004) used a serial-like visual search and did not obtain a PS effect. It is likely that there, too, participants simply used an exhaustive search, and thus there was no RT effect. It appears that the PS effects obtained with the RT measure in focused attention tasks (e.g., Devue \& Brédart, 2008; Gronau et al., 2003, 2005, 2009) and in search tasks that do not call for an exhaustive search (Exp. 1 of the present study) will not be obtained in search tasks that call for exhaustive search (Exp. 2 of the present study; Harris et al., 2004).

As a whole, our results clearly support the hypothesis that PS stimuli affect performance and elicit ORs only when presented within the focus of attention. As detailed earlier (see also Gronau et al., 2003), attentional factors can explain all of the discrepancies between previous studies that addressed this question. Studies in which interference by unattended PS distractors has been found did not use paradigms in which attention was tightly monitored. Our study provides direct evidence for the role of attention as a mediator for interference from an irrelevant PS distractor, and by extension indicates that the PS effect in all of the above studies is indeed due to the role of attention.

One potential objection to our study is its implicit assumption that participants are able to process the words in all conditions, and that the lack of a PS effect has specifically to do with attentional filtering of contents that are irrelevant for the task. Alternatively, one may claim that the lack of a PS effect has to do with a lack of perceptual processing of the words. Recent research by Lien, Ruthruff, Kouchi, and Lachter (2010) appears to support this possibility. These authors used a priming paradigm and repeatedly demonstrated that word primes affect performance when they are presented within the attentional focus, but not when they are presented outside the attentional focus. However, while there seems to be good evidence that unattended words do not prime subsequent performance, there is equally good evidence that unattended words cause semantic interference (e.g., Gronau et al., 2003, 2009;
Lachter, Ruthruff, Lien, \& McCann, 2008). The reason for the differential effects of words in priming and interference paradigms is not entirely clear (see Lien et al., 2010, for an extensive discussion of this issue). More relevant for the present purposes, our study used an interference paradigm, and as just discussed, there is good evidence in such paradigms that unattended words do affect performance when they are task relevant, which means that words can be processed in such situations. Within this context, our findings suggest that PS but task-irrelevant words do not affect performance.

Finally, the present findings may shed further light on the conditions for attention capture. There are disagreements in the attention literature concerning the nature of stimuli that capture attention. Yantis and Jonides (1984) suggested that abrupt onsets (i.e., stimuli that abruptly appear in a location that previously did not contain an object) capture attention by bottom-up processes. Others have suggested that singletons (i.e., stimuli that include a unique feature) are also able to capture attention (e.g., Theeuwes, 1991). Others yet (e.g., Folk et al., 1992) claimed that there is no bottom-up attention capture, and that only stimuli that are part of the task attentional set can capture attention. Our finding, that attention was not captured by the illuminated stimuli in the control experiment, seems to support the notion that the luminance manipulation per se does not lead to attention capture and that top-down processes determine whether attention will be captured by these stimuli. Our study, however, was not designed for this purpose, and consequently, it is possible that the specific conditions used in our experiments prevented attention capture by the singleton. For example, the two levels of luminance that were used in our study were arbitrary, and the difference between the high- and low-luminance stimuli might not have been sufficiently large to be effective on its own for attention capture.

In conclusion, we aimed to create a situation in which attention would be maximally monitored, in order to validly examine the ability of PS information to affect performance when presented within versus outside the focus of attention. Our approach was to directly manipulate attention, and to do so while obtaining an empirical index for its operation that would allow us to infer that attention was indeed involved. We found that when these conditions were met, by using an endogenous manipulation of attention, PS distractors did not affect performance when presented outside the focus of attention, but only when presented within the focus of attention. This finding further supports the previous claim that appearance within or outside the focus of attention is a critical mediator in determining whether or not PS information will affect performance. 
Author Note This research was funded by grants from the Israel Science Foundation to G.B.S. and A.C. We thank Ori Cohen, Noa Erlich, Arit Gliksohn, Michal Preiss, and Maya Zuckerman for their assistance in this research.

\section{References}

Arnell, K. M., Shapiro, K. L., \& Sorensen, R. E. (1999). Reduced repetition blindness for one's own name. Visual Cognition, 6, 609-635.

Ben-Shakhar, G., \& Gati, I. (1987). Common and distinctive features of verbal and pictorial stimuli as determinants of psychophysiological responsivity. Journal of Experimental Psychology: General, 116, 91-105.

Broadbent, D. E. (1958). Perception and communication. New York: Oxford University Press.

Bundesen, C. (1990). A theory of visual attention. Psychological Review, 97, 523-547.

Bundesen, C., Kyllingsbaek, S., Houmann, K. J., \& Jensen, R. M. (1997). Is visual attention automatically attracted by one's own name? Perception \& Psychophysics, 59, 714-720.

Cohen, A., \& Shoup, R. (1997). Perceptual dimensional constraints on response selection processes. Cognitive Psychology, 32, 128181

Conway, A. R., Cowan, N., \& Bunting, M. F. (2001). The cocktail party phenomenon revisited: The importance of working memory capacity. Psychonomic Bulletin \& Review, $8,331-335$

Devue, C., \& Brédart, S. (2008). Attention to self-referential stimuli: Can I ignore my own face? Acta Psychologica, 128, 290-297. doi:10.1016/j.actpsy.2008.02.004

Duncan, J., \& Humphreys, G. W. (1989). Visual search and stimulus similarity. Psychological Review, 96, 433-458.

Eriksen, B. A., \& Eriksen, C. W. (1974). Effects of noise letters upon the identification of a target letter in a nonsearch task. Perception \& Psychophysics, 16, 143-149.

Eriksen, C. W., \& Yeh, Y. Y. (1985). Allocation of attention in the visual field. Journal of Experimental Psychology: Human Perception and Performance, 11, 583-597.

Folk, C. L., Remington, R. W., \& Johnston, J. C. (1992). Involuntary covert orienting is contingent on attentional control settings. Journal of Experimental Psychology: Human Perception and Performance, 18, 1030-1044.

Folk, C. L., Remington, R. W., \& Wright, J. H. (1994). The structure of attentional control: Contingent attentional capture by apparent motion, abrupt onset, and color. Journal of Experimental Psychology: Human Perception and Performance, 20, 317-329. doi:10.1037/0096-1523.20.2.317

Gronau, N., Ben-Shakhar, G., \& Cohen, A. (2005). Behavioral and physiological measures in the detection of concealed information. Journal of Applied Psychology, 90, 147-158.

Gronau, N., Cohen, A., \& Ben-Shakhar, G. (2003). Dissociations of personally significant and task-relevant distractors inside and outside the focus of attention: A combined behavioral and psychophysiological study. Journal of Experimental Psychology: General, 132, 512-529. doi:10.1037/0096-3445.132.4.512

Gronau, N., Cohen, A., \& Ben-Shakhar, G. (2009). Distractor interference in focused attention tasks is not mediated by attention capture. Quarterly Journal of Experimental Psychology, 62, 1685-1695. doi:10.1080/17470210902811223

Gronau, N., Sequerra, N., Cohen, N., \& Ben-Shakhar, G. (2006). The effect of novel distractors on performance in focused attention tasks: A cognitive-psychophysiological approach. Psychonomic Bulletin \& Review, 13, 570-575.
Harris, C. R., \& Pashler, H. E. (2004). Attention and the processing of emotional words and names: Not so special after all. Psychological Science, 15, 171-178.

Harris, C. R., Pashler, H. E., \& Coburn, N. (2004). High-priority affective stimuli and visual search. Quarterly Journal of Experimental Psychology, 57, 1-31.

Jonides, J. (1981). Voluntary versus automatic control over the mind's eye's movements. In J. Long \& A. Baddeley (Eds.), Attention and performance $I X$ (pp. 187-203). Hillsdale: Erlbaum.

Kawahara, J., \& Yamada, Y. (2004). Does one's name attract visual attention? Visual Cognition, 11, 997-1017.

Kornblum, S., Hasbroucq, T., \& Osman, A. (1990). Dimensional overlap: Cognitive basis for stimulus-response compatibility-a model and taxonomy. Psychological Review, 97, 253-270. doi:10.1037/0033-295X.97.2.253

Laarni, J., Koljonen, M., Kuistio, A. M., Kyrolainen, S., Lempiainen, J., \& Lepisto, T. (2000). Images of a familiar face do not capture attention under conditions of inattention. Perceptual and Motor Skills, 90, 1216-1218.

Lachter, J., Forster, K. I., \& Ruthruff, E. (2004). Forty-five years after Broadbent: Still no identification without attention. Psychological Review, 111, 880-913.

Lachter, J., Ruthruff, E., Lien, M. C., \& McCann, R. S. (2008). Is attention needed for word identification? Evidence from the Stroop paradigm. Psychonomic Bulletin \& Review, 15, 950-955.

Lien, M. C., Ruthruff, E., Kouchi, S., \& Lachter, J. (2010). Even frequent and expected words are not identified without spatial attention. Attention, Perception, \& Psychophysics, 72, 973-988.

Mack, A., \& Rock, I. (1998). Inattentional blindness. Cambridge: MIT.

Moray, N. (1959). Attention in dichotic listening: Affective cues and the influence of instructions. Quarterly Journal of Experimental Psychology, 11, 56-60.

Pashler, H. (1998). The psychology of attention. Cambridge: MIT.

Posner, M. I. (1980). Orienting of attention. Quarterly Journal of Experimental Psychology, 32, 3-25.

Rauschenberger, R. (2003). Attentional capture by auto- and allocues. Psychonomic Bulletin \& Review, 10, 814-842.

Raymond, J. E., Shapiro, K. L., \& Arnell, K. M. (1992). Temporary suppression of visual processing in RSVP task: An attentional blink? Journal of Experimental Psychology: Human Perception and Performance, 18, 849-860.

Shapiro, K. L., Caldwell, J., \& Sorensen, R. E. (1997). Personal names and the attentional blink: A visual "cocktail party" effect. Journal of Experimental Psychology: Human Perception and Performance, 23, 504-514.

Sokolov, E. N. (1963). Perception and the conditioned reflex. New York: Macmillan.

Sternberg, S. (1969). The discovery of processing stages: Extensions of Donders' method. In W. G. Koster (Ed.), Attention and performance II (pp. 276-315). Amsterdam: North-Holland.

Stroop, J. R. (1935). Studies of interference in serial verbal reactions. Journal of Experimental Psychology, 18, 643-662. doi:10.1037/ 0096-3445.121.1.15

Theeuwes, J. (1991). Cross-dimensional perceptual selectivity. Perception \& Psychophysics, 50, 184-193.

Treisman, A. M. (1960). Contextual cues in selective listening. Quarterly Journal of Experimental Psychology, 12, 242-248.

Treisman, A. M., \& Gelade, G. (1980). A feature-integration theory of attention. Cognitive Psychology, 12, 97-136.

Wolfe, J. M., Cave, K. R., \& Franzel, S. L. (1989). Guided search: An alternative to the feature integration model for visual search. Journal of Experimental Psychology: Human Perception and Performance, 15, 419-433.

Wolford, G., \& Morrison, F. (1980). Processing of unattended visual information. Memory \& Cognition, 8, 521-527. 
Wood, N., \& Cowan, N. (1995). The cocktail party phenomenon revisited: How frequent are attention shifts to one's own name in an irrelevant auditory channel? Journal of Experimental Psychology: Learning, Memory, and Cognition, 21, 255-260.

Yantis, S., \& Johnston, J. (1990). On the locus of visual selection: Evidence from focused attention tasks. Journal of
Experimental Psychology: Human Perception and Performance, 16, 135-149.

Yantis, S., \& Jonides, J. (1984). Abrupt visual onsets and selective attention: Evidence from visual search. Journal of Experimental Psychology: Human Perception and Performance, 10, 601-621.

Zimny, G. H., \& Schwabe, L. W. (1965). Stimulus change and habituation of the orienting response. Psychophysiology, 2, 103-114. 